\title{
Bioinformatic comparison of Kunitz protease inhibitors in Echinococcus granulosus sensu stricto and E. multilocularis and the genes expressed in different developmental stages of E. granulosus s.s.
}

Hui Zhang ${ }^{1,2+}$, Mengxiao Tian ${ }^{1,2+}$, Wenjing Qi ${ }^{1,2}$, Juan Wu ${ }^{1,2}$, Huajun Zheng ${ }^{3}$, Gang Guo $^{1,2}$, Liang Zhang ${ }^{3}$, Shiwanthi L. Ranasinghe ${ }^{4}$, Donald P. McManus ${ }^{4}$, Jun $\mathrm{Li}^{1,2^{*}}$ and Wenbao Zhang ${ }^{1,2^{*}}$

\begin{abstract}
Background: Cystic and alveolar echinococcosis caused by the tapeworms Echinococcus granulosus sensu stricto (s.s.) and E. multilocularis, respectively, are important zoonotic diseases. Protease inhibitors are crucial for the survival of both Echinococcus spp. Kunitz-type inhibitors play a regulatory role in the control of protease activity. In this study,we identified Kunitz-type domain protease inhibitors(KDPIs) present in the genomes of these two tapeworms and analyzed the gene sequences using computational, structural bioinformatics and phylogenetic approaches to evaluate the evolutionary relationships of these genes. Hi-seq transcriptome analysis showed that $E$. granulosus s.s. KDPIs were differentially expressed in the different developmental stages. We validated some of the genes expressed in adult worm, protoscolex and cyst germinal membrane of E. granulosus s.s. and E. multilocularis by quantitative PCR.

Results: A total of 19 genes from E. multilocularis and 23 genes from E. granulosus s.s. were predicted to be KDPIs with the most containing a single Kunitz-domain. A maximum likelihood method phylogenetic tree indicated that the $E$. granulosus s.s. and E. multilocularis Kunitz domain peptides were divided into three branches containing 9 clusters. The ratio of positively charged residues and neutral residues are different between E. multilocularis and E. granulosus s.s. KDPIs. We also found that E. multilocularis had higher percentage of sequences containing signal peptides (17/19, $89.47 \%)$ than that of E. granulosus s.s. (14/23, 60.87\%). Transcript analysis showed all the E. granulosus s.s. KDPI genes were expressed differentially in four developmental stages of the worm. Transcription analysis showed that 9 KDPIs (including EG_07244,EGR_08716 and EGR_10096) were highly upregulated in adult worm, and 2 KDPIs (EG_09268 and EG_09490) were highly expressed in the cyst germinal membrane. Quantitative gene expression analysis(qPCR) of
\end{abstract}

*Correspondence: 1742712944@qq.com; wenbaozhang2013@163.com

${ }^{+}$Hui Zhang and Mengxiao Tian contributed equally to this work.

${ }^{2}$ State Key Laboratory of Pathogenesis, Prevention and Treatment

of High Incidence Diseases in Central Asia, WHO-Collaborating Centre

for Prevention and Care Management of Echinococcosis, Xinjiang Medical

University, The First Affiliated Hospital of Xinjiang Medical University,

830054 Urumqi, Xinjiang, China

Full list of author information is available at the end of the article

(c) The Author(s) 2021. Open Access This article is licensed under a Creative Commons Attribution 4.0 International License, which permits use, sharing, adaptation, distribution and reproduction in any medium or format, as long as you give appropriate credit to the original author(s) and the source, provide a link to the Creative Commons licence, and indicate if changes were made. The images or other third party material in this article are included in the article's Creative Commons licence, unless indicated otherwise in a credit line to the material. If material is not included in the article's Creative Commons licence and your intended use is not permitted by statutory regulation or exceeds the permitted use, you will need to obtain permission directly from the copyright holder. To view a copy of this licence, visit http://creativecommons.org/licenses/by/4.0/. The Creative Commons Public Domain Dedication waiver (http://creativeco mmons.org/publicdomain/zero/1.0/) applies to the data made available in this article, unless otherwise stated in a credit line to the data. 
four genes confirmed the expression of these genes. EGR_08716 and its homologous gene (EmuJ_001137000) were highly and specifically expressed in adult worms of the two worms.

Conclusions: A total 19 and 23 KDPIs were identified in the genomes of E. multilocularis and E. granulosus s.s. , respectively. The differential expression of these KDPIs in different stages may indicate their different roles in the different hosts. The difference in characterization of KDPIs may be associated with the different pathology of metacestode stage of these two parasites.

Keywords: Kunitz inhibitors, E. multilocularis, E. granulosus sensu stricto, Bioinformatic analysis, Stage expression

\section{Background}

Cyst echinococcosis (CE) and alveolar echinococcosis (AE) are both medically and economically important diseases caused by the metacestode stages of Echinococcus granulosus sensu stricto (s.s.) and E. multilocularis respectively. The diseases impact on hundreds of millions of people in Asia, Europe, American and Africa [1]. The control and treatment of echinococcosis are difficult. High frequency of dosing dogs with the drug praziquantel has played a key role in the control of the disease [2,3], but undertaking the control measure is challenging in remote areas. A vaccine against adult worms in dogs is urgently needed [4].

The life-cycle of these two tapeworms involves four developmental stages including adult worm, oncosphere, cyst and protoscolex present in their definitive and intermediate hosts. The major definitive and intermediate hosts of E. granulosus s.s. are dogs and sheep respectively, whereas, the natural definitive and intermediate hosts of E. multilocularis are fox/wolf and rodent small mammals respectively. Humans are the intermediate hosts of these two tapeworms. The survival of these tapeworms relies on evading host immune responses and avoiding attack by proteases; this is especially important for the adult parasites which reside in the gastrointestinal duct, a location where high concentration of proteases are present which are harmful and toxic for the worms.

Eukaryote proteases including serine (trypsin/chymotrypsin-like), cysteine (thiol) and aspartic (pepsin/ cathepsin/rennin) proteases play a fundamental role in the regulation of protein function. Their functions and activities are controlled largely by protease inhibitors which play crucial roles in the regulation of proteases involved in a range of biological processes system including cell proliferation, inflammation, cell homeostasis and immune mechanism [5-7]; protease inhibitors act mainly through the control of potentially disadvantageous, excessive or inopportune proteolytic activity. Protease inhibitors including aspartic, cysteine, metallo, serine, and threonine inhibitors are super-families based on their similarities at the amino acid sequence level and tertiary structure [8]. Similarities in primary structure and tertiary structure support the common ancestry of many protease inhibitor families.

Kunitz-type domain protease inhibitors (KDPIs) are an important type of protease inhibitor and belong to the I2 family of protease inhibitors $[8,9]$. These inhibitors contain at least one cysteine-rich peptide chain (Kunitz-type domain) with $\alpha$ and $\beta$ sheets. The Kunitz domain consists of around 60 amino acids including six conserved cysteine residues forming three disulphide bridges in a characteristic pattern (C1-C6, C2-C4, and C3-C5) [9, 10], which stabilize these inhibitors. KDPIs have been characterized from animals and plants $[9,11]$ including helminths [12-14]. A previous study described eight genes (EgKU1-EgKU8) isolated from E. granulosus s.s. protoscoleces treated with pepsin/H (+) [15]. We previously cloned and characterized two E. granulosus s.s. KDPIs, EgKI-1(EG_08721 (GenBank: EUB56407.1)) and EgKI-2 (EG_07242 (GenBank: EUB57880.1)) [14]. EgKI-1 is highly expressed in the oncosphere (egg) stage and is a potent chymotrypsin and neutrophil elastase inhibitor that binds calcium and reduced neutrophil infiltration in a local inflammation model. EgKI-2 is highly expressed in adult worms, it is a potent inhibitor of trypsin and is a potential vaccine candidate against echinococcosis in dogs [14]. Beyond these, other E. granulosus s.s. and E. multilocularis KDPIs have been received little attention.

In the present study, we identified all KDPI sequences predicted in the E. granulosus s.s. and E. multilocularis genomes and used computerized programs to characterize these Kunitz domain protease inhibitors. We show that the majority of E. granulosus s.s. KDPIs were differentially expressed in different life cycle stages and some have a range of GO numbers indicating these inhibitors likely function in different ways in the tapeworm's development.

\section{Results}

General characterizations of Kunitz domain protease inhibitors

InterproScan and Motif scan identified 19 and 23 genes encoding KDPIs from the E. multilocularis and E. granulosus s.s. genomes, respectively (Table 1). The KDPI family has a typical Kunitz domain of about 60 amino acids in 
Table 1 Summary of physiological and biological characteristics of Kunitz protease inhibitors in E. multilocularis and $E$. granulosus s.s.

\begin{tabular}{lll}
\hline Species & E. multilocularis & E. granulosus s.s \\
\hline KDPIs/single KDPIs & $19 / 16$ & $23 / 21$ \\
Number of amino acids & 333.47 & 269.96 \\
Molecular weight (Da) & 37153.2 & 30117.02 \\
Isoelectric points & 7.44 & 7.84 \\
No. of trans-domain (\%) & $4(21.05)$ & $5(21.74)$ \\
No. of cysteine in Kunitz domain & 5.63 & 5.65 \\
No. of cysteine in the protein & 24.89 & 18.13 \\
Instability index & 45.17 & 47.36 \\
Stable protein (No/yes) & $12 / 7$ & $15 / 8$ \\
Aliphatic index & 72.35 & 71.37 \\
Grand average of hydropathicity & -0.22 & -0.22 \\
Signal peptides (\%) & $17(89.47)$ & $14(60.87)$ \\
No. of Kunitz Motifs & 1.79 & 1.17 \\
En-t-In(T/C) & $9 / 2$ & $9 / 4$ \\
\hline
\end{tabular}

Note: KDPIs, Kunitz domain protease inhibitors; No. of aa, number of amino acids; No. of tran-domain, percentage of containing transmembrane domains; Aver of cysteine, average of cysteine per sequence; Aliphatic indexes; GRSVY, hydropathic index; En-t-In (T/C), enzyme targeting inhibitors, trypsin inhibitors $(\mathrm{T})$ or chymotrypsin inhibitors $(\mathrm{C})$

There are significant differences of signal peptides between $E$. multilocularis and E. granulosus s.s. KDPIs $\left(X^{2}=13.544, P<0.01\right)$

size (Fig. 1) with a special secondary structure formed by three disulphide bonds or bridges (Additional file 3: Fig. S1). The echinococcal Kunitz domains contain an average of 52.85 aa (range 47-55 aa) with the majority comprising 53 aa (Fig. 1).

Among these KDPIs, E. multilocularis has 16 KDPIs containing a single Kunitz domain with the complete proteins having sizes of 75-534 aa. There are 3 proteins containing multi-domains with a maximum 8 Kunitz domains (EmuJ_001181950) in size ranging from 610 to 2425 aa. E. granulosus s.s. has 21 single Kunitz domain KDPIs of 75-976 aa in size, and 2 multiple domain KDPIs sized from 878 to 1540 aa. The molecular weights of these KDPIs range from $8.34 \mathrm{kDa}$ to $266.77 \mathrm{kDa}$ with isoelectric points from 4.52 to 10.52 (Additional file 1: Table S1). The majority of single Kunitz domain proteins comprise less than 100 amino acid (Additional file 1: Table S1).

We used the instability index to estimate the stability of the KDPIs. An instability value $>40$ is an unstable protein. The index value representing rigidity/flexibility of each peptide varied (26.51-94.41). The average value was 45.17 for E. multilocularis and 47.37 for E. granulosus s.s. , suggesting the peptides may be flexible. The analysis showed that E. multilocularis has 12 unstable and 7 stable KDPIs and E. granulosus s.s. has 15 unstable and 8 stable KDPIs (Additional file 1: Table S1).

Sequence analysis showed that these echinococcal KDPI sequences contain a high percentage of hydrophobic residues including alanine $(\mathrm{A})$, valine $(\mathrm{V})$, leucine $(\mathrm{L})$ and isoleucine (I). The hydropathic index (Grand Average of Hydropathy: GRAVY) for E. granulosus s.s. and E. multilocularis are $-0.223 \pm 0.333$ and $-0.222 \pm 0.312$, respectively. Aliphatic indexes (AI) are $71.37 \pm 14.08$ and $72.36 \pm 13.75$ for E. granulosus s.s. and E. multilocularis, respectively (Table 1 and Additional file 1: Table S1).

The average numbers of negatively charged residues (Asp+Glu) are 38.42 and 28.91, accounting for $8.84 \%$ and 9.13\% of E. multilocularis and E. granulosus s.s. KDPIs, respectively. There are 35.16 and 30.70 positively charged residues (Arg+Lys) in the E. multilocularis and E. granulosus s.s. KDPIs accounting for $12.73 \%$ and $12.23 \%$ of the total amino acids. Neutral amino acid residues are 259.89 and 210.35 aa on average and account for $78.42 \%$ and $78.63 \%$ of the KDPIs in E. multilocularis and E. granulosus s.s., respectively (Table 1 and Additional file 1: Table S1).

The average aliphatic indexes are 72.36 (51.54-89.78) and 71.37 (49.44-100.73) for the E. multilocularis and E. granulosus s.s. KDPIs, respectively. The hydropathicity indexes of E. multilocularis and E. granulosus s.s. KDPIs are -0.222 (ranging from -0.978 to 0.340 ) and -0.223 (ranging from -0.996 to 0.371 ) for respectively. The results indicate that the KDPIs in both parasites are likely hydrophilic proteins (Table 1 and Additional file 1 : Table S1).

E. multilocularis and E. granulosus s.s. have 4 and 5 KDPIs containing transmembrane regions, respectively, and $78.94 \%$ and $78.26 \%$ of the E. multilocularis and E. granulosus s.s. KDPIs are extracellular (Table 1), which matches the GO analysis (Table 2 and Additional file 2: Table S2), indicating that the most KDPIs may involve host and parasite interface responses. The TopPred program indicated that 4 E. multilocularis and 5 E. granulosus s.s. KDPIs are located on the cytoplasm (Additional file 1: Table S1) with others, including 15 Em-KDPI sequences and $18 \mathrm{Eg}$-KDPIs, being extracellular.

(See figure on next page.)

Fig. 1 Alignment and clustering of E. multilocularis and E. granulosus s.s. Kunitz-type domain protease inhibitors. These inhibitor protein sequences are compared with the homologue sequences (blocked in gray background) Homo sapiens including P48307(Hsa), P05067(Hsa), P12111(Hsa) and O95925(Hsa), Ovis aries (NP_001009291.1(Oar)), Bos taurus(1BTH_Q(Bt)), Schistosoma mansoni (Smp_147730 (Sm)), Stichodactyla haddoni(B1B5I8(Sha)), Stichodactyla helianthus (3OFW_A(She)), Trinorchestia longiramus(KAF2343884(TI)), Haplopelma schmidti (P68425(Hsc)). "En”, "“T", "C" and "/" represent enzyme and inhibitor of trypsin, chymotrypsin, and nonprediction, respectively 


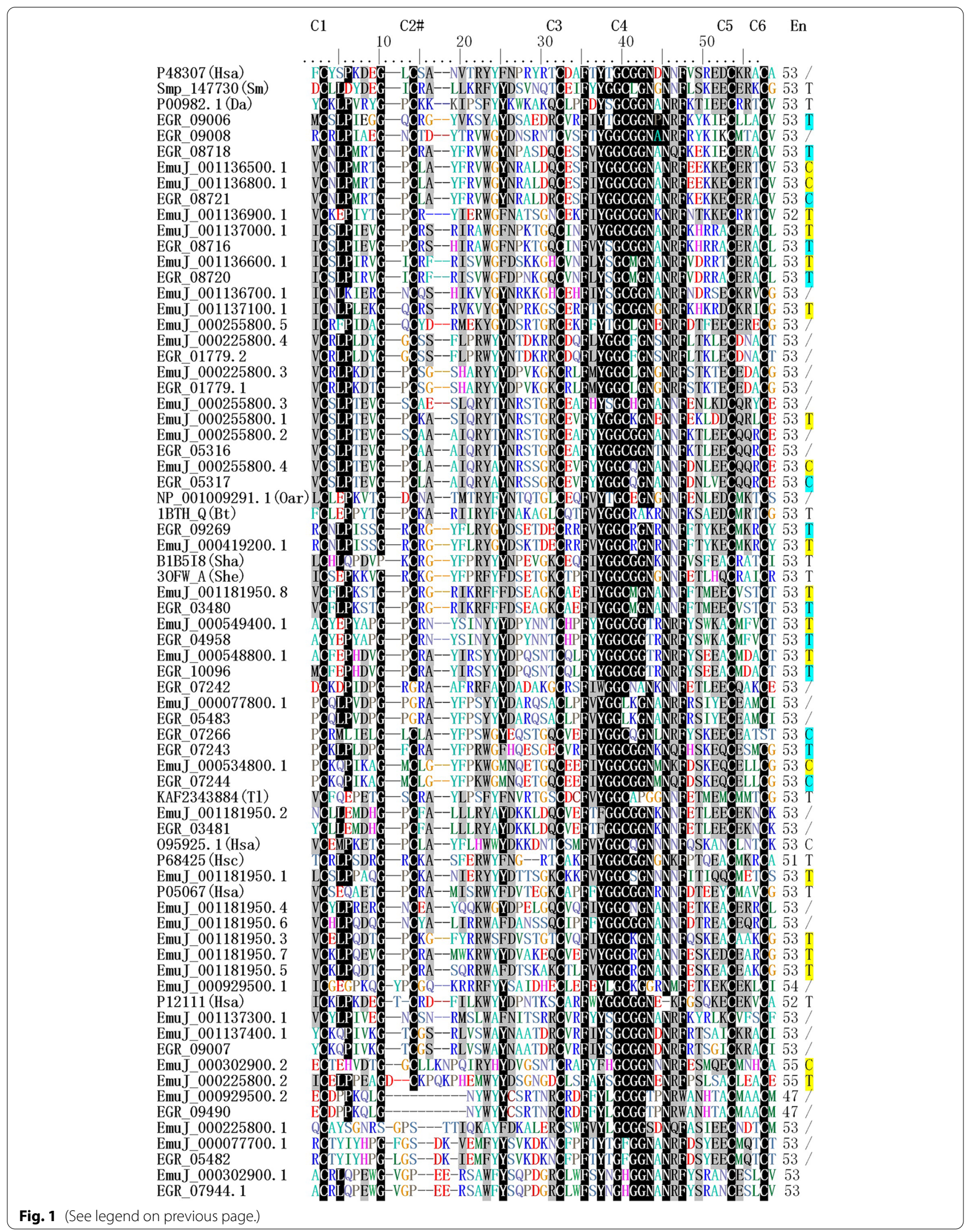


Table 2 The expression of Kunitz-type domain protease inhibitors in four stages of E. granulosus s.S. showing Hi-seq reads

\begin{tabular}{|c|c|c|c|c|c|c|c|c|}
\hline Gene ID & Adt & Onc & PSC & CGM & Seq. Description & $\begin{array}{l}\text { Seq. } \\
\text { Lth }\end{array}$ & \#GOs & GOs \\
\hline EGR_07244 & 217 & 0 & 1 & 10 & serine protease inhibitor & 106 & 1 & F: serine-type endopeptidase inhibitor activity \\
\hline EGR_08716 & 155 & 0 & 0 & 0 & kunitz-type protease inhibitor 3-like & 84 & 3 & $\begin{array}{l}\text { F: peptidase inhibitor activity; F: protein binding; } \\
\text { P: transforming growth factor beta receptor } \\
\text { signaling pathway }\end{array}$ \\
\hline EGR_10096 & 70 & 0 & 3 & 4 & kunitz domain-containing & 98 & 42 & $\begin{array}{l}\text { C: cytoplasmic vesicle; P: apoptosis; P: neuro- } \\
\text { muscular process controlling balance; P: iono- } \\
\text { tropic glutamate receptor signaling pathway; P: } \\
\text { regulation of epidermal growth factor receptor } \\
\text { activity; F: receptor binding; P: regulation o }\end{array}$ \\
\hline EGR_07242 & 46 & 0 & 1 & 5 & serine protease inhibitor & 83 & 7 & $\begin{array}{l}\text { F: peptidase inhibitor activity; C: nematocyst; F: } \\
\text { ion channel inhibitor activity; F: potassium chan- } \\
\text { nel inhibitor activity; F: serine-type endopepti- } \\
\text { dase inhibitor activity; C: extracellular region; P: } \\
\text { pathogenesis }\end{array}$ \\
\hline EGR_07243 & 35 & 0 & 2 & 5 & wap four-disulfide core domain $6 \mathrm{~b}$ & 75 & 2 & $\begin{array}{l}\text { C: cytoplasm; F: serine-type endopeptidase } \\
\text { inhibitor activity }\end{array}$ \\
\hline EGR_03480 & 24 & 0 & 1 & 5 & four-domain proteases inhibitor & 88 & 1 & F: peptidase inhibitor activity \\
\hline EGR_09490 & 17 & 2 & 14 & 51 & spon-1 protein & 976 & 3 & $\begin{array}{l}\text { F: serine-type endopeptidase inhibitor activity; } \\
\text { C: proteinaceous extracellular matrix; C: extracel- } \\
\text { lular region }\end{array}$ \\
\hline EGR_03481 & 16 & 0 & 8 & 2 & trypsin inhibitor & 242 & 4 & $\begin{array}{l}\text { F: peptidase inhibitor activity; P: multicellular } \\
\text { organismal process; F: extracellular matrix struc- } \\
\text { tural constituent; C: proteinaceous extracellular } \\
\text { matrix }\end{array}$ \\
\hline EGR_08720 & 10 & 0 & 0 & 0 & $\begin{array}{l}\text { kunitz bovine pancreatic trypsin inhibitor } \\
\text { domain protein }\end{array}$ & 84 & 4 & $\begin{array}{l}\text { F: serine-type endopeptidase inhibitor activity; F: } \\
\text { peptidase activity; F: peptidase inhibitor activity; } \\
\text { C: extracellular region }\end{array}$ \\
\hline EGR_07944 & 9 & 0 & 22 & 14 & $\begin{array}{l}\text { kunitz bovine pancreatic trypsin inhibitor } \\
\text { domain protein }\end{array}$ & 539 & 2 & C: extracellular region; F: hydrolase activity \\
\hline EGR_09269 & 8 & 0 & 0 & 1 & tissue factor pathway inhibitor 2-like & 92 & 2 & $\begin{array}{l}\text { F: extracellular matrix structural constituent; C: } \\
\text { proteinaceous extracellular matrix }\end{array}$ \\
\hline EGR_05317 & 7 & 0 & 19 & 16 & Kunitz-like protease inhibitor precur & 1540 & & \\
\hline EGR_08721 & 6 & 108 & 0 & 0 & $\begin{array}{l}\text { serine protease inhibitor- with kunitz and wap } \\
\text { domains } 1\end{array}$ & 79 & 1 & C: acrosomal vesicle \\
\hline EGR_04958 & 5 & 0 & 1 & 2 & $\begin{array}{l}\text { elegans protein partially confirmed by tran- } \\
\text { script evidence }\end{array}$ & 135 & 2 & $\begin{array}{l}\text { F: serine-type endopeptidase inhibitor activity; P: } \\
\text { epidermis development }\end{array}$ \\
\hline EGR_05483 & 5 & 12 & 0 & 2 & secreted protein with kunitz & 191 & 2 & C: extracellular region; F: hydrolase activity \\
\hline EGR_09007 & 4 & 0 & 0 & 0 & $\begin{array}{l}\text { mechanosensory abnormality family member } \\
\text { (mec-1) }\end{array}$ & 86 & 2 & $\begin{array}{l}\text { P: extracellular structure organization; P: mecha- } \\
\text { nosensory behavior }\end{array}$ \\
\hline EGR_09008 & 3 & 0 & 0 & 0 & acp24a4 & 102 & 2 & C: extracellular region; F: peptidase activity \\
\hline EGR_01779 & 1 & 0 & 13 & 3 & isoform g & 878 & 1 & F: hydrolase activity \\
\hline EGR_05482 & 1 & 2 & 0 & 0 & $\begin{array}{l}\text { kunitz bovine pancreatic trypsin inhibitor } \\
\text { domain containing protein }\end{array}$ & 130 & 1 & C: extracellular region \\
\hline EGR_07266 & 1 & 0 & 0 & 3 & serine protease inhibitor & 129 & 3 & $\begin{array}{l}\text { F: binding; F: serine-type endopeptidase inhibi- } \\
\text { tor activity; C: extracellular region }\end{array}$ \\
\hline EGR_05316 & 0 & 0 & 3 & 2 & kunitz-type protease inhibitor 3-like & 239 & 1 & F: peptidase inhibitor activity \\
\hline EGR_08718 & 0 & 2 & 0 & 0 & kunitz domain-containing & 144 & 4 & $\begin{array}{l}\text { F: peptidase activity; F: serine-type endopepti- } \\
\text { dase inhibitor activity; F: peptidase inhibitor } \\
\text { activity; C: extracellular region }\end{array}$ \\
\hline EGR_09006 & 0 & 0 & 0 & 0 & single kunitz protease inhibitor & 89 & 5 & $\begin{array}{l}\text { F: peptidase activity; F: serine-type endopepti- } \\
\text { dase inhibitor activity; F: peptidase inhibitor } \\
\text { activity; C: extracellular region; P: multicellular } \\
\text { organismal development }\end{array}$ \\
\hline
\end{tabular}


Signal peptide analysis showed that there are 17/19(89.47\%) E. multilocularis KDPIs having an 18-26 amino acid (aa) signal peptide and 2/19(10.53\%) KDPIs without. In contrast, E. granulosus s.s. has 14/23 (60.87\%) KDPIs containing signal peptide sequences and 9/23 (39.13\%) KDPIs without (Table 1). There is significant difference of signal peptides between $E$. multilocularis and E. granulosus s.s. KDPIs $\left(\mathrm{X}^{2}=13.544, P<0.01\right.$, Table 1).

\section{Cluster and phylogenetic analysis of Kunitz protease inhibitors}

Multiple sequence alignment (Fig. 1) and phylogenetic analysis (Fig. 2) of the amino acid sequences were used to infer the evolutionary relationships between the $E$. multilocularis and E. granulosus s.s. KDPIs and to make a comparison with other species. Figure 2 shows the different evolutionary distances of the genes containing single Kunitz domain of the KDPIs using the maximum likelihood method. The maximum likelihood tree indicated that these echinococcal KDPIs were divided into three branches with 9 clusters. One branch contains several Echinococcus KDPIs such as EGR_3480, EGR_3481, EGR_05316 and EGR_07242 are close to the KDPIs from human, sheep, cattle and other species (Fig. 2 and Additional file 6: Fig. S4). Whereas other two branches are relatively Echinococcus unique (Fig. 2).

It showed that two KDPIs of EGR_05482 and EmuJ_000077700.1 from E. granulosus s.s. and E. multilocularis shared a high homology with mamba venom toxin P00982.1 and P00981 from Dendroaspis angusticeps and Dendroaspis polylepis respectively (Fig. 2), indicating these echinococcal KDPIs may have similar pathophysiological functions of mamba venom blocking ion channels and membrane receptors.

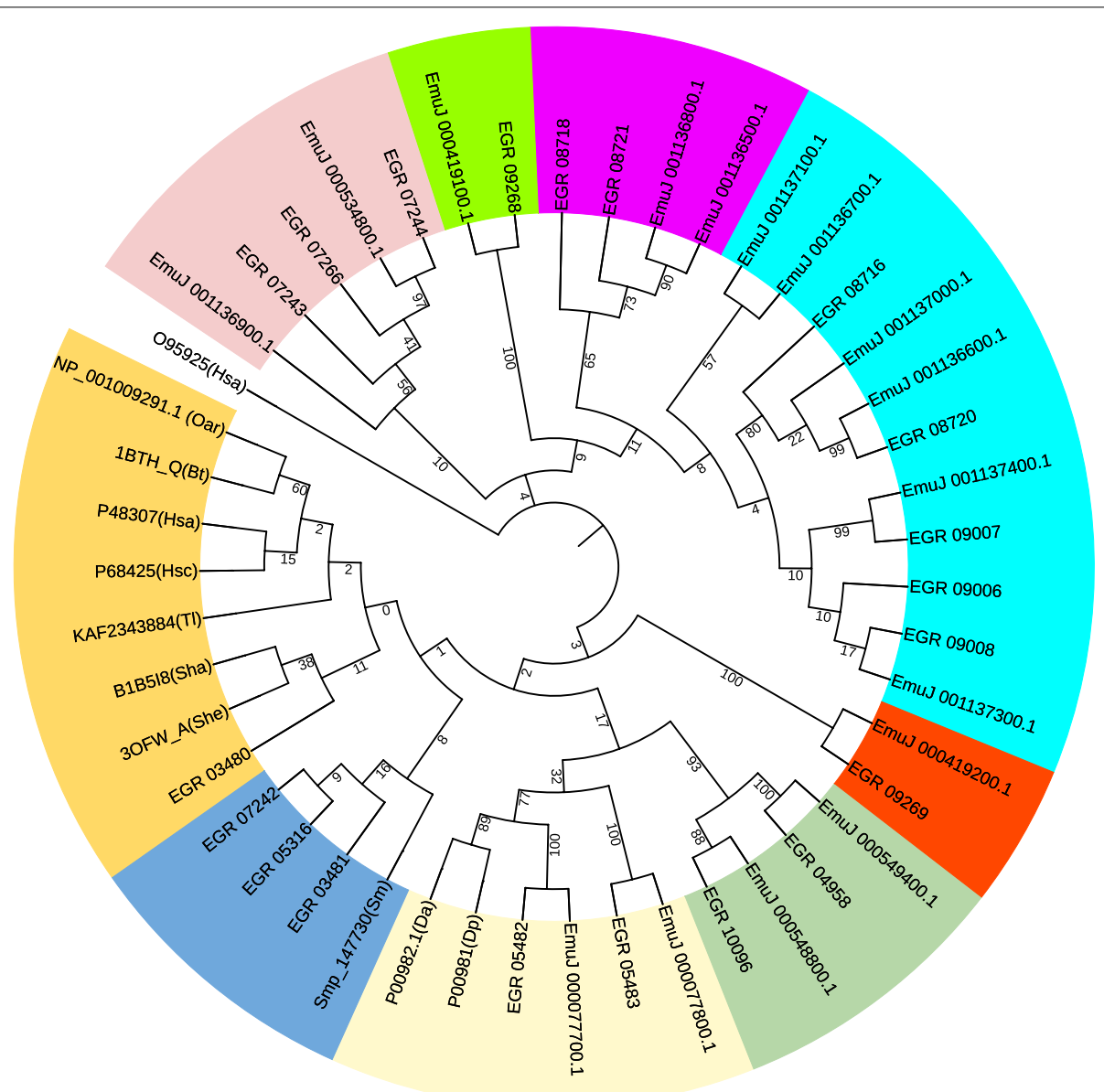

Fig. 2 Phylogram constructed to compare the sequences of E. granulosus s.s.- and E. multilocularis-KDPIs with KDPIs from Bos taurus, Homo sapiens and other species. These inhibitor protein sequences are also compared with the sequences from Dendroaspis including Dendroaspis angusticeps (P00982.1(Da)), Dendroaspis polylepis (P00981(Dp)). The phylogenetic tree was constructed using MEGA 7.0 and Interactive Tree of Life iTOL v6 with the maximum likelihood method. Bootstrapping analysis was performed and the bootstrap values from 1000 replicate iterations are shown on the nodes. The clades are represented by different color 


\section{Comparison of KDPI genes predicated from the $E$.} granulosus S.s. and E. multilocularis genomes

We compared the KDPI genes predicted from the genomes of E. granulosus s.s. and E. multilocularis and found that some genes are species-specific. E. multilocularis does not have homologues of $E$. granulosus s.s. sequences EG_07242, EG_07266, EG_07243, EG_09006 and EG_09008; whereas EmuJ_001136700.1 and EmuJ_001137100.1 are specific to E. multilocularis (Additional file 5: Fig. S3).

The specificity of a protease inhibitor against a protease is mainly determined by the nature of the amino acid residue at position $\mathrm{P} 1$ of its active site. It has been shown that $\operatorname{Lys}(\mathrm{K})$ and $\operatorname{Arg}(\mathrm{R})$ mutants of bovine pancreatic trypsin inhibitor (BPTI) bind to bovine trypsin about $10^{5}$-fold stronger than BPTI with P1 Tyr(T) [16]. In addition, it has been shown that typical trypsin inhibitors have $\operatorname{Arg}(\mathrm{R})$ or $\operatorname{Lys}(\mathrm{K})$ at $\mathrm{P} 1$, and chymotrypsin inhibitors have Leu (L) or Met (M) at the P1 position [17]. Therefore, the sequence analysis shows that the $E$. multilocularis has $10 \mathrm{KDPIs}$ containing $\mathrm{R}$ at $\mathrm{P} 1$ and E. granulosus s.s. has $11 \mathrm{KDPIs}$ containing $\mathrm{R}$ at $\mathrm{P} 1$, which belong to typical trypsin inhibitors. Furthermore, the two tapeworms have 3 or 4 sequences containing $L$ at P1 respectively, which are chymotrypsin inhibitors (Fig. 1; Table 1).

\section{$2 \mathrm{D}$ and $3 \mathrm{D}$ structure of Kunitz domain protease inhibitors}

The majority of E. multilocularis and E. granulosus s.s. single KDPIs are small proteins sized $16-\mathrm{kDa}$ and contain a relatively high percentage of Lys and Arg residues at the $\mathrm{C}$-terminus. Like most Kunitz domain protease inhibitors, the Em- and Eg-KDPIs contain a conserved Kunitz type sequence with 6 cysteine residues forming three disulphide bridges (C1-C6, C2-C4 and C3-C5) (Additional file 3: Fig. S1) and these bridges play a key role in the formation of the $2 \mathrm{D}$ and $3 \mathrm{D}$ structure of these KDPIs. For the single Kunitz domain sequences, the secondary structure prediction revealed $19.01-52.71 \%$ and $18.6-60.35 \%$ of $\alpha$-helix and random coil structures in Eg-KDPIs, followed by extended strands and $\beta$-turn structure, accounting for $13.1-26.67 \%$ and $1.89-10.84 \%$ respectively. Em-KDPIs $\alpha$-helix and random coil structures account for $19-40.45 \%$ and $32.5-55.99 \%$ of the protein sequence respectively, followed by extended strands and $\beta$-turn, accounting for $8-36.25 \%$ and $0-10.71 \%$ (Table 3).

It is accepted that there is a close relationship between the structure and function of a protein. Therefore, we used SWISS-MODEL to predict 3D structures based on the homology modeling of KDPI templates from PDB (protein database) including single and multiple Kunitz domain proteins (Fig. 3 and Additional file 4: Fig. S2).
Three-dimensional structure analysis showed that a single Kunitz domain sequence with three disulphide bridges has a similar structure containing an $\alpha$-helix and random coils with similar structures (Fig. 3). The structure of some single Kunitz domain sequences lose the second cysteine $(\mathrm{C} 2)$ which may impact the $3 \mathrm{D}$ structure of these KDPIs. (Fig. 1; Table 1).

\section{Expression of E. granulosus s.s. KDPIs in different developmental stages}

To estimate expression of the KDPIs, Hi-seq techniques were employed to obtain the transcript reads of these genes from total RNA from each of 4 developmental stages of E. granulosus s.s. . The transcript read information was published in a previous paper of ours [18]. The transcriptome analysis showed that these Kunitz peptides were differentially expressed in the different developmental stages of E. granulosus s.s. (Table 2). All the inhibitors, except EG_09006, were expressed in one or 4 stages of $E$. granulosus s.s. with some being highly and differentially expressed in one or two stages. Transcription analysis showed that 9 KDPIs including EGR_03480, EGR_03481, EGR_07242, EGR_07243, EG_07244,EGR_08716, EGR_08720, EGR_09269 and EGR_10096 were highly up-regulated in adult worm, and two KDPIs (EG_09268 and EG_09490) were highly expressed in the cyst germinal membrane. Sequence analysis showed that some of these adult worm up-regulated genes are extracellular including EG_03480, EG_07242, EG_08716, EG_08720, EG_09490 and EG_10096, and some are intracellular such as EG_03481, EG_07243 and EG_07244 (Table 2 and Additional file 1: Table S1).

EG_08716 is an extracellular protease inhibitor and has 42 predicted GOs, including cytoplasmic vesicle for neuromuscular process controlling balance, ionotropic glutamate receptor signaling pathway, regulation of the activity of epidermal growth factor receptor and synapse, regulation of mitotic cell cycle and translation and cellular copper and calcium ion homeostasis (Additional file 1: Table S1). The expression analysis indicated that this gene may play an important role in adult worm development and against host protease attack. It is interesting that EG_07244 is predicted having endopeptidase activity, indicating that the protein has two functions, as a peptidase and as a protease inhibitor in adult worms. This needs to be identified. EG_08721 is an extracellular inhibitor and was differentially highly expressed in the oncosphere compared with the other stages, indicating this protease inhibitor plays an important role in oncosphere biology, the only stage for primarily infection and EG_08721 may play an important role in oncosphere against host protease attack which may be a candidate for vaccine development. 
Table 3 The secondary structure prediction of the single Eg-KDPIs and Em-KDPIs

\begin{tabular}{|c|c|c|c|c|}
\hline Accession number & Alpha helix(aa/\%) & Extended strand(aa/\%) & Beta turn(aa/\%) & Random coil(aa/\%) \\
\hline EGR_03480 & $32 / 36.36$ & $19 / 21.59$ & $3 / 3.41$ & $34 / 38.64$ \\
\hline EGR_03481 & $46 / 19.01$ & $57 / 23.55$ & $19 / 7.85$ & $120 / 49.59$ \\
\hline EGR_04958 & $33 / 24.44$ & $28 / 20.74$ & $11 / 8.15$ & $63 / 46.67$ \\
\hline EGR_05316 & $92 / 38.17$ & $54 / 22.41$ & $17 / 7.05$ & $78 / 32.37$ \\
\hline EGR_05482 & $54 / 41.54$ & 18/13.85 & $8 / 6.15$ & $50 / 38.46$ \\
\hline EGR_05483 & $66 / 34.55$ & $41 / 21.47$ & $10 / 5.24$ & $74 / 38.74$ \\
\hline EGR_07242 & $25 / 30.12$ & $13 / 15.66$ & $9 / 10.84$ & $36 / 43.37$ \\
\hline EGR_07243 & $20 / 26.67$ & $20 / 26.67$ & $5 / 6.67$ & $30 / 40$ \\
\hline EGR_07244 & $41 / 38.68$ & $23 / 21.7$ & $2 / 1.89$ & $40 / 37.74$ \\
\hline EGR_07266 & $68 / 52.71$ & $29 / 22.48$ & $8 / 6.2$ & 24/18.6 \\
\hline EGR_07944.1 & $138 / 25.6$ & $91 / 16.88$ & $34 / 6.31$ & $276 / 51.21$ \\
\hline EGR_08716 & $31 / 36.9$ & $11 / 13.1$ & $3 / 3.57$ & $39 / 46.43$ \\
\hline EGR_08718 & $41 / 28.47$ & $34 / 23.61$ & $8 / 5.56$ & $61 / 42.36$ \\
\hline EGR_08720 & $25 / 29.76$ & $18 / 21.43$ & $4 / 4.76$ & $37 / 44.05$ \\
\hline EGR_08721 & $35 / 44.3$ & $13 / 16.46$ & $3 / 3.8$ & $28 / 35.44$ \\
\hline EGR_09006 & $28 / 31.46$ & $22 / 24.72$ & $9 / 10.11$ & $30 / 33.71$ \\
\hline EGR_09007 & $23 / 26.74$ & $13 / 15.12$ & $7 / 8.14$ & $43 / 50$ \\
\hline EGR_09008 & $43 / 42.16$ & $22 / 21.57$ & $7 / 6.86$ & $30 / 29.41$ \\
\hline EGR_09269 & $37 / 40.22$ & $15 / 16.3$ & $4 / 4.35$ & $36 / 39.13$ \\
\hline EGR_09490 & $203 / 20.8$ & $145 / 14.86$ & $39 / 4.0$ & $589 / 60.35$ \\
\hline EGR_10096 & $20 / 20.41$ & $23 / 23.47$ & $3 / 3.06$ & $52 / 53.06$ \\
\hline EmuJ_000077700.1 & $65 / 30.95$ & $42 / 20$ & $17 / 8.1$ & $86 / 40.95$ \\
\hline EmuJ_000077800.1 & $33 / 24.26$ & $39 / 28.68$ & $10 / 7.35$ & $54 / 39.71$ \\
\hline EmuJ_000302900.1 & $128 / 23.97$ & $76 / 14.23$ & $31 / 5.81$ & 299/55.99 \\
\hline EmuJ_000419200.1 & $37 / 40.22$ & $15 / 16.30$ & $0 / 0.00$ & $40 / 43.48$ \\
\hline EmuJ_000534800.1 & $24 / 32.00$ & $6 / 8.00$ & $5 / 6.67$ & $40 / 53.33$ \\
\hline EmuJ_000548800.1 & $23 / 23.23$ & $21 / 21.21$ & $4 / 4.04$ & $51 / 51.52$ \\
\hline EmuJ_000549400.1 & $19 / 19.00$ & $27 / 27.00$ & $8 / 8.00$ & $46 / 46.00$ \\
\hline EmuJ_001136500.1 & $36 / 40.45$ & $16 / 17.98$ & $2 / 2.25$ & $35 / 39.33$ \\
\hline EmuJ_001136600.1 & $31 / 36.90$ & $13 / 15.48$ & $6 / 7.14$ & $34 / 40.48$ \\
\hline EmuJ_001136700.1 & $20 / 25.64$ & $18 / 23.08$ & $8 / 10.26$ & $32 / 41.03$ \\
\hline EmuJ_001136800.1 & $36 / 40.45$ & $16 / 17.98$ & $2 / 2.25$ & $35 / 39.33$ \\
\hline EmuJ_001136900.1 & $21 / 23.33$ & $17 / 18.89$ & $4 / 4.44$ & $48 / 53.33$ \\
\hline EmuJ_001137000.1 & $26 / 30.95$ & $16 / 19.05$ & $4 / 4.76$ & $38 / 45.24$ \\
\hline EmuJ_001137100.1 & $22 / 26.19$ & $20 / 23.81$ & $9 / 10.71$ & $33 / 39.29$ \\
\hline EmuJ_001137300.1 & $20 / 25$ & $29 / 36.25$ & $5 / 6.25$ & $26 / 32.5$ \\
\hline EmuJ_001137400.1 & $26 / 30.59$ & 16/18.82 & $4 / 4.71$ & $39 / 45.88$ \\
\hline
\end{tabular}

Note: aa/\%, number/percentage of amino acids in each secondary structure

Although we activated PSC with pepsin, only three KDPIs (EG_01779, EG_05317 and EG_07944) were slightly elevated in this stage. Importantly, we found that EG_09268 and EG_09490 were highly expressed in the cyst germinal membrane and the proteins expressed by these genes may be potential targets for drug development.

To validate the expression of these genes, a qPCR assay was employed to quantify the transcript levels of 4 genes of E. granulosus s.s. (EGR_08716, EGR_07244,
EGR_07944 and EGR_09490), which confirmed the expression in cyst germinal membranes, protoscoleces and adult worm stages of the tapeworm. We also identified the homologous KDPI genes expression from $E$. multilocularis (EmuJ_001137000, EmuJ_000534800, EmuJ_000302900, and EmuJ_000929500) in three stages of the tapeworm (Fig. 4). Only one gene, EmuJ_001137000 showed a similar expression pattern as E. granulosus s.s. EG_08716, is highly and specifically expressed in the adult worms of $E$. multilocularis (Fig. 4). 
a

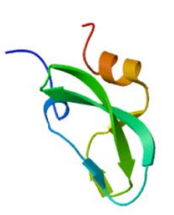

53
4
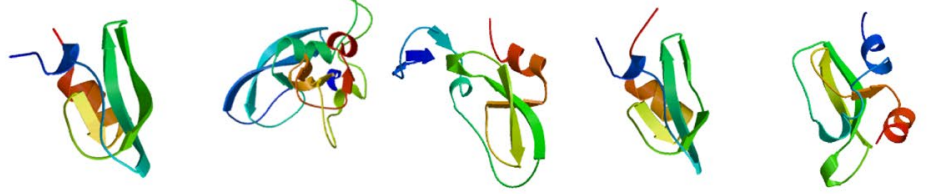

EG_03480

EG_03481

EG_04958

EG_05316

EG_05482

EG_09006

EG_09269

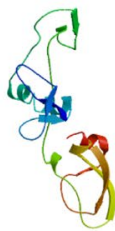
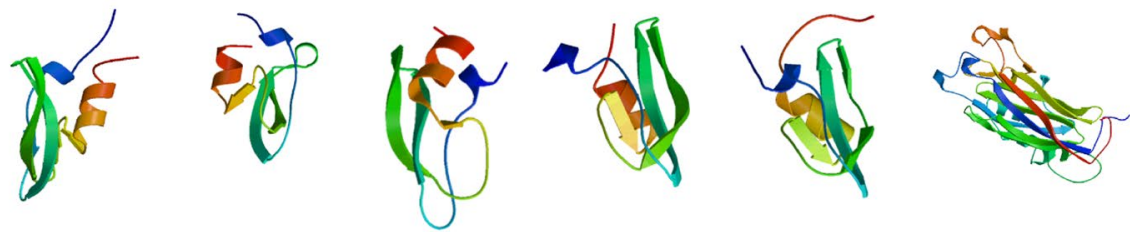

EG_05483

EG_07242

EG_07243

EG_07244

EG_07266

EG_09007

EG_09490
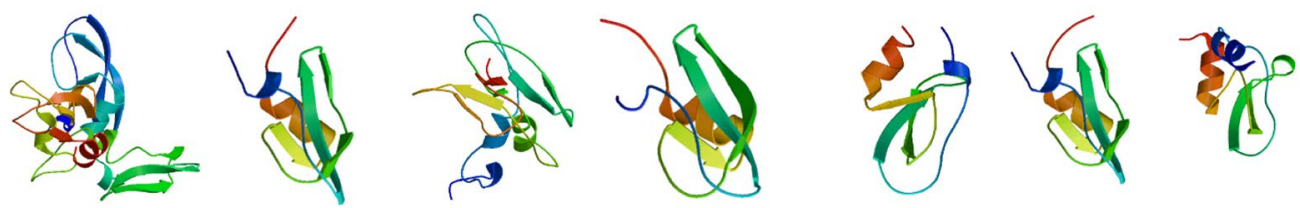

EG_07944

EG_08716

EG_08718

EG_08720

EG_08721

EG_09008

EG_10096

b

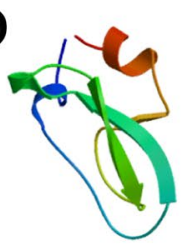

EmuJ_001136500

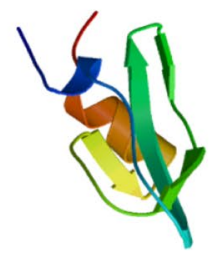

EmuJ_001137000

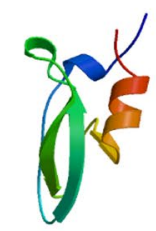

EmuJ_000077800

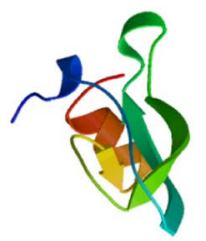

EmuJ_001136600

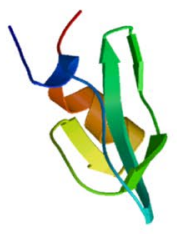

EmuJ 001137100

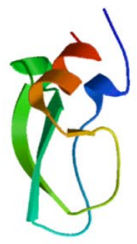

EmuJ_000534800.1

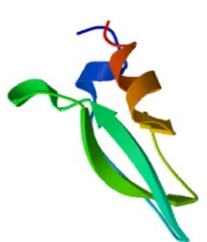

EmuJ_001136700

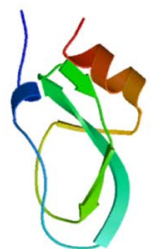

EmuJ_001137300

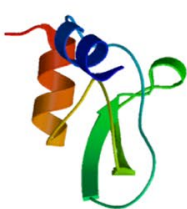

EmuJ_000548800

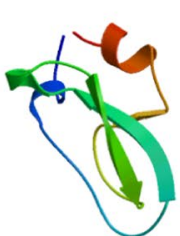

EmuJ_001136800

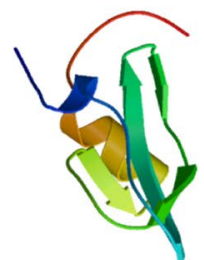

EmuJ_001137400

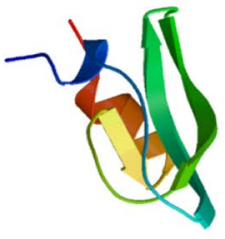

EmuJ_000549400

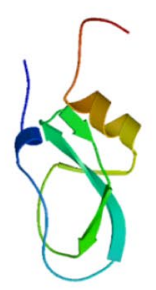

EmuJ_001136900

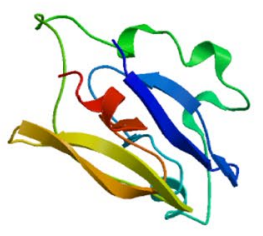

EmuJ 000077700

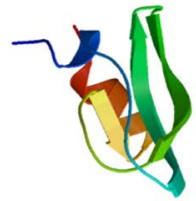

EmuJ_000419200

Fig. 3 Three dimensional structures of single Kunitz domain protease inhibitors in E. granulosus s.S. and E. multilocularis using SWISS-MODEL. a Structures of single Kunitz domain protease inhibitors in E. granulosus s.s. . b Structures of single Kunitz domain protease inhibitors in E. multilocularis 
Other three genes were different from those homologous genes of E. granulosus s.s. , which were highly expressed in protoscoleces of this tapeworm (Fig. 4). The qPCR showed that E. multilocularis adult worm EmuJ_001137000 was 4.43 folds higher than EGR_08716 in the adult worms of E. granulosus s.s. .

\section{Discussion}

KDPIs occur in almost all living organisms from bacteria to plants and animals. Kunitz peptides show diverse biological activities including inhibition of proteases and/or blocking or modulating ion channels. Helminth parasites have been reported expression KDPIs [19], such as in Schistosoma mansoni [12], S. japonicum [20] Fasciola hepatica [21, 22], Ancylostoma caninum [23], Ancylostoma ceylanicum [24], and Steinernema carpocapsae [25]. Beside their role in inhibition of proteases such as pancreatic elastase, neutrophil elastase, chymotrypsin and trypsin, KDPIs play an important role in helminth's immune evasion and development, modulating the inflammatory responses, especially impairing Th1/Th17-associated inflammation response and reduction of neutrophil recruitment [19]. In this study, based on the genomic information available for E. granulosus s.s. and E. multilocularis we identified 23 and 19 KDPIs, respectively. These genes were differential expressed in different developmental stages, indicating these KDPIs may play different role in both parasite development and regulation the interface responses between parasites and hosts.

A remarkable difference between the larval stages of $E$. multilocularis and E. granulosus s.s. is the difference in the lesion pathology in the intermediate hosts. The metacestode of E. multilocularis is a tumor-like, infiltrating structure consisting of many small vesicles embedded in the stroma of connective tissue. The continual growth of parasite vesicles in a proliferative style causes damage of liver tissues, which results in a high mortality of AE patients. In contrast, E. granulosus s.s. cysts develop in internal organs (mainly liver and lungs) of humans and other intermediate hosts as unilocular fluid-filled bladders with clear edge between cyst and host tissue. CE causes mortality in very few patients and there is a relatively good prognosis after surgical removal of the cystic lesion. Contrastingly, $\mathrm{AE}$ causes severe damage to the liver and patients require extensive treatment with albendazole to prevent relapse. However, little is known about the molecular mechanisms underpinning biological differences between the two parasites and the diseases they cause.
The differential expression of these KDPI genes between E. granulosus s.s. and E. multilocularis may be associated with the differences in pathology caused by the metacestodes of the two species. It would be informative to determine whether these genes play a role in determining the different pathologies resulting from infection by the two cestodes in their intermediate hosts.

Signal peptide analysis showed that $89.47 \%$ of $E$. multilocularis KDPIs contain a signal peptide compared to only $60.87 \%$ of E. granulosus s.s. KDPIs containing signal peptide sequences. It may indicate that the proportion of secretion protein in E. multilocularis KDPIs is relatively higher than that of E. granulosus s.s. KDPIs. It is not known whether the high percentage KDPIs of E. multilocularis containing signal peptides is associated with the virulent pathology of AE lesion.

E. granulosus s.s. has 5 genes EG_07242, EG_07266, EG_07243, EG_09006 and EG_09008, that E. multilocularis does not have. Whereas, these two genes, EmuJ_001136700.1 and EmuJ_001137100.1 are only existed in E. multilocularis genome. These differentially presented genes may play a role in the difference of pathology between the two parasites.

Gastrointestinal helminths survive in an environment containing proteases and these parasites must have mechanisms to control protease activation. Therefore, Kunitz domain inhibitors are important for parasite survival, especially intestinal dwelling helminth parasites, to counteract protease attack. Two Echinococcus stages, the oncosphere and adult worm, are found in the gastrointestinal duct. The oncosphere is activated in the stomach and penetrates through the intestinal wall before being passed into the internal organs, whereas the adult worm spends its whole life in the gastrointestinal duct which contains high concentrations of proteases such as pepsin, trypsin and chymotrypsin. We previously showed that two KDPIs, EgKI-1(EG_08721) and EgKI-2 (EG_7242) function as protease inhibitors. EgKI-1 (also has accession number EUB56407.1) is highly expressed in the oncosphere and EgKI-2 (GenBank: EUB57880.1) is highly expressed in the adult worm [14]. These KDPIs are differentially expressed and stage-specifically protect E. granulosus s.s. from protease attack [13]. In this study, we showed that 11 out 23 Eg-KDPIs were highly expressed in adult worms. These Eg-KDPIs likely protect against protease attacks in the gut during adult worm development. EG_05483 and EG_08721 were relatively highly expressed in oncospheres, suggesting their expressed products might be potential vaccine

(See figure on next page.)

Fig. 4 Transcription levels of KDPIs (EGR_08716, EGR_07244, EGR_07944, EGR_09490, EmuJ_001137000, EmuJ_000534800, EmuJ_000302900, and EmuJ_000929500) in different life cycle stages of E. granulosus s.s. and E. multilocularis. Bars indicate the mean value \pm standard deviation (SD) of three individual experiments. Differences among different groups were analyzed by one-way ANOVA and Two-tailed Student's t test. "Adult", "PSC", and "CGM" represent adult worm, protoscoleces, and cyst germinal membrane three stages of the tapeworm, respectively 
I E. granulosus

A

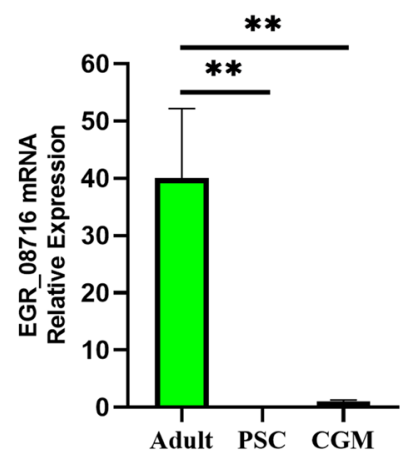

B

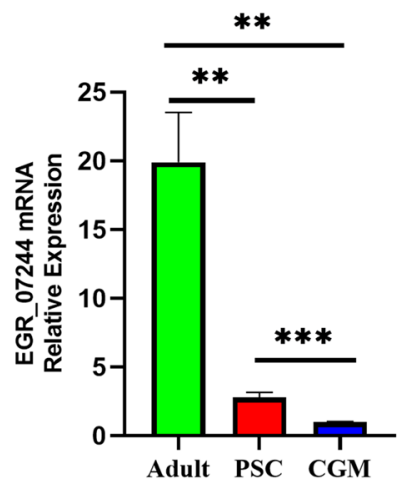

C

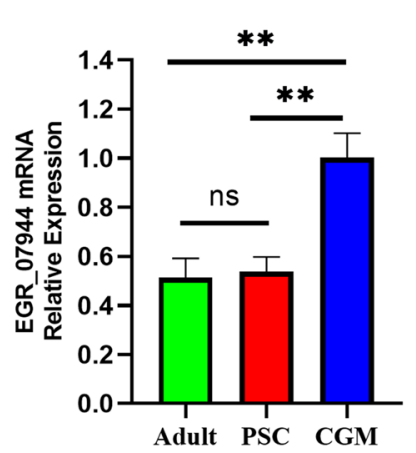

D

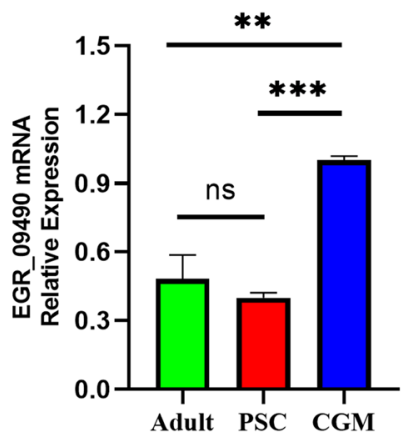

II E. multilocularis

E

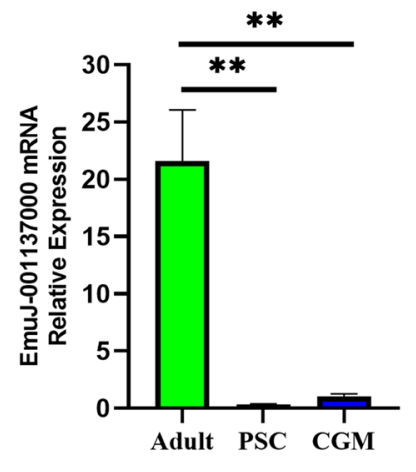

F

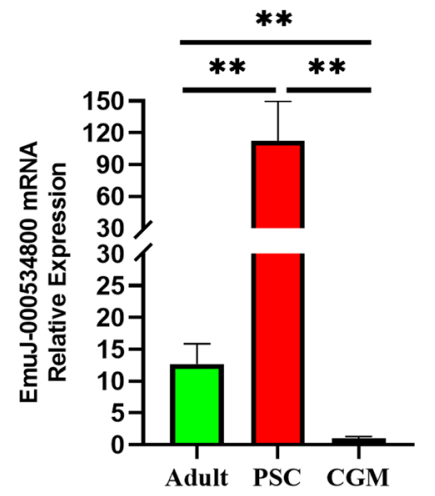

G

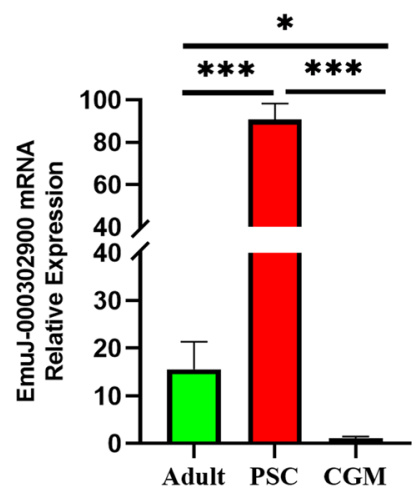

H

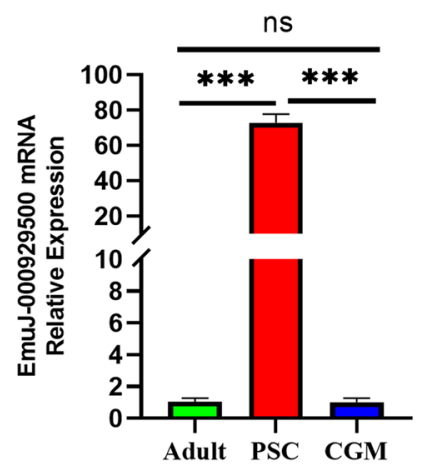


candidates against primary infection in the intermediate hosts of E. granulosus s.s. .

We did not find any KDPIs that were differentially and highly expressed in protoscoleces in this study, although a previous study described a multigene family of eight (EgKU1-EgKU8) secreted Kunitz proteins from E. granulosus s.s. protoscoleces preferentially expressed by pepsin/H (+)-treated worms [15].

The secondary structures of proteins, especially the $\alpha$-helix and $\beta$-strands play key roles in molecular function, cell stability, mechanical signaling, and tissue constitution as random coils are easily folded and exposed to the protein surface [26]. The basic structure of a Kunitz peptide domain contains a typical sequence with six highly conserved cysteine residues connecting three disulphide bridges (C1-C6, C2-C4 and C3-C5) which stabilizes the protein structure. Among the disulphide bridges, the $\mathrm{C} 1-\mathrm{C} 6$ and $\mathrm{C} 3-\mathrm{C} 5$ bridges are required for the maintenance of native confirmation [27], whereas the C2-C4 bond stabilizes the folded structure [28]. We found 10 sequences had lost the \#2 cysteine, including 5 from E. granulosus s.s., indicating no $\mathrm{C} 2-\mathrm{C} 4$ bridge in these proteins. The reduction of disulfide bonds may affect the stability of the protein. It is not known whether these 5 proteins formed different bridges impacting on the function of these KDPIs, indicating that these genes may have a different functional role.

Hydrophilicity analysis showed that the Em- and Eg-KDPIs have high hydrophobicity, which is a typical characteristic of membrane proteins. The transmembrane regions consist of 20 hydrophobic amino acids, which could have an anchoring effect on cell membranes.

We previously showed that EgKI-1 is highly expressed in the oncosphere, indicating this protein helps protect this stage from digestion by trypsin, chymotrypsin and pancreatic elastase before it penetrates the intestinal wall.

In this study, qPCR results showed that 3 out 4 KDPI genes of E. multilocularis homologous to those E. granulosus s.s. KDPIs were expressed in different pattern in three stages of this tapeworm including adult worm, protoscolex and cyst germinal membrane. It will be interesting to identify whether the expression of KDPIs is associated with the pathological difference in both intermediate hosts and definitive hosts of these two tapeworms.

It is predicted that EgKU-1 and EgKU-4 functionally blocks voltage-dependent potassium channels (Kv). The similar sequences of E. multilocularis EmuJ_001136700 and EmuJ_001137100(Additional file 8: Fig. S6), may likely function as a Kv blocker [29], which may be a clue for designing drug targets against alveolar echinococcosis. In addition, Eudiplozoon nipponicum EnKT1 (a $\mathrm{KDPI}$ ) is an antihemorrhagic snake venom factor-like protein which exhibited a higher activity against plasmin and Factor Xa which can act as a C3 and C5 convertase and impaired both haemostasis and complement activation [30]. The different expression of similar KDPIs in E. granulosus s.s. and E. multilocularis may be associated with the difference in cellular pathology of these two echinococcal diseases. It needs to be uncovered in future studies.

It is shown that parasite KDPIs with immunomodulatory activity can be vaccine candidates. Fasciola hepatica FhKTM decreased dendritic cell activation and may be involved in the immune evasion mechanisms of the parasite [21]. FhKTM induced mice protection against F. hepatica challenge by preventing liver damage and improving survival, likely through eliciting potent IFNgamma and IL-17 A with high levels of antigen-specific IgG1, IgG2a, and IgA serum antibodies [31]. The KDPI EGR_05316 of E. granulosus s.s. has sililar sequences with Smp_147730 of S. mansoni [12, 32]. Two KDPIs EGR_05483 and EmuJ_000077800.1 of E. granulosus s.s. and E. multilocularis have similar sequences with FhKT1.1, FhKT1.2 and FhKT2 of F. hepatica [33] (Additional file 7: Fig. S5).

A study showed that Schistosoma mansoni Kunitz peptides were highly protective in vaccinated BALB/c mice in terms of reduction in recovery of adult females (89 91\%) and in the numbers of eggs trapped in the livers (77 81\%) and guts (57 77\%) of mice [32]. In addition, SmKI-1 showed 23 33\% of reductions in adult worm [13]. EGR_03481, EGR_07242 and EGR_05316 of E. granulosus s.s. KDPIs have very similar sequences with Smp_147730 (Fig. 2), this indicates that these KDPIs of Echinococcus likely can be candidates for vaccination against echinococcosis.

\section{Conclusions}

In conclusion, based on whole genome analysis, 19 and 23 Kunitz domain protease inhibitors were identified in E. multilocularis and E. granulosus s.s. . The differential expression of these KDPIs in different developmental stages of E. granulosus s.s. suggests that they may have different functions in regulation of host immune responses. The difference in characterization of KDPIs may be associated with the different pathology of metacestode stage of these two parasites. These should be further illuminated to determine their roles in echinococcal development and interface interactions between host and the tapeworms and such information may provide new insights for the prevention and treatment of cystic and alveolar echinococcosis. 


\section{Materials and methods}

\section{Identification of $E$. granulosus s.s. and $E$.} multilocularis Kunitz domain sequences

The E. granulosus s.s. and E. multilocularis genomes were previously completed by the Chinese National Human Genome Centre in Shanghai (CHGC) and the Wellcome Sanger Institute, United Kingdom in 2013 [18, 34]. Genome data is available from http://www.sanger. ac.uk/ resources/downloads/helminths/ (E. multilocularis, E. granulosus). The complete genome annotation is available at www.genedb.org.

E. granulosus s.s. , E. multilocularis and Fasciola hepatica sequence data reported in this manuscript are accessible at WormBase ParaSite and the corresponding accession numbers indicated in Additional file 9: Table S3. E. multilocularis data have been deposited in the National Center for Biotechnology Information (NCBI) under the project accession number PRJEB122 and their Genome Assembly accession number is GCA_000469725.3. E. granulosus s.s. data have been deposited in NCBI (BioProject numbers: PRJEB121 and PRJNA182977) and their Genome Assembly accession numbers were GCA_000469785.1 and ASM52419v1. Based on the DNA genomic sequences, 11,325 and 10,429 genes were predicted for E. granulosus s.s. , and E. multilocularis, respectively. The InterProScan program (https://www.ebi.ac.uk /interpro/result/InterProScan/) and Motif scan (https://myhits.sib.swiss/ cgi-bin /motif_scan) were used to identify the Kunitz domain protease inhibitor (KDPI) sequences.

\section{Physiological/biochemical characters}

The physiological/biochemical characters of KDPIs including molecular weight, isoelectric point and instability index were predicted using the ProtParam online software (http://web.Expasy.org/protparam/). Signal peptides were predicted with the SignalP 5.1 Server (http:// www.cbs.dtu.dk/services/SignalP/). Post-translational modification sites were identified by MotifScan (http:// hits.isb-sib.ch/cgi-bin/motif_scan/).

The conservative structural domain of each KDPI was predicted using the Conserved Domain program (http://www.ncbi.nlm.nih.gov/cdd/); their subcellular localization was predicted using ProtCompv. 9.0 (http://linux1.softberry.com/berry. phtml?topic $=p r$ otcompan\&group $=$ programs \&subgroup $=$ proloc $/)$. Transmembrane regions were predicted by TMPred (http://embnet.vital-it.ch/cgibin/TMPRED__ form_ parser) and TopPred 1.10 (http://mobyle.pasteur.fr/ cgi-bin/portal.py?\# forms::toppred). The hydrophilicity plot was predicted by ProtScale (http://web. expasy.org/protscale/). The secondary structures of KDPIs were predicted using SOPMA (https:// npsa-prabi.ibcp.fr/cgi-bin/npsa_automat.pl?page $=$ npsa_sopma/). These genes were annotated to the Gene Ontology (GO) database for biological process (BP), molecular function (MF), and cellular component (CC) using Blast2GO PRO (https://www.blast 2go.com/).

Three-dimensional (3D) structures of KDPIs were constructed using the automated modeling program within the online service SWISS-MODEL. The 3D models of KDPIs were assessed by Verify_3D (http://services.mbi. ucla.edu/Verify_3D/).

The multiple sequence alignment was analyzed and ordered by Clustal omega (http://www.ebi.ac.uk/Tools/ $\mathrm{msa} / \mathrm{clustalo} /$ ) and then visually edited with BioEdit software v7.1.3. The phylogenetic tree was constructed by MEGA version 7 (http://www.megasoftware.net/) and Interactive Tree of Life iTOL v6 (https://itol.embl.de) with the maximum likelihood method. Bootstrapping analysis was performed and the bootstrap values which display support values (1000 bootstraps) are shown on the nodes. The clades are represented by different color. The complete KDPI protein sequences of single domain KDPIs were used for phylogenetic tree analysis.

\section{Expression of Kunitz domain inhibitors in E. granulosus s.s. and E. multilocularis}

Transcript reads were obtained for each of the KDPI genes expressed in the adult worm, oncosphere, protoscolex and cyst (cyst germinal membrane) of $E$. granulosus s.s. using Hiseq techniques as described [18]. Based on these data, the expression of 8 KDPI genes from the E. multilocularis and E. granulosus S.s. expression was validated for adult worm, protoscolex and cyst stages using quantitative reverse transcription PCR (qRT-PCR).

\section{Total RNA extraction and cDNA synthesis}

E. granulosus s.s. protoscoleces were collected from the cysts of sheep livers collected in slaughterhouses in Urumqi, Xinjiang, China. E. multilocularis protoscoleces were obtained by Mongolia gerbils maintained in the animal laboratory of the First Affiliated Hospital of Xinjiang Medical University. Adult worms of E. granulosus s.s. $(35$ days-old) and E. multilocularis (25 days-old) were provided by the Institute of Veterinary Research, Xinjiang Academy of Animal Sciences.

Isolated protoscoleces were washed 10 times with saline and stored at $-80{ }^{\circ} \mathrm{C}$ until used. TRIzol reagent (Invitrogen, USA) was used for extraction of total RNA according to the manufactory's instructions. RNA concentration was determined by a spectrophotometer (NanoDrop 2000, Thermo Scientific, USA) at $260 \mathrm{~nm}$ and the purity of RNA was considered satisfactory if the 
Table 4 Sequences of forward and reverse primers for the genes analysed by qPCR

\begin{tabular}{|c|c|c|}
\hline Gene & Primer sequences $\left(5^{\prime}-3^{\prime}\right)$ & Product size \\
\hline EGR_08716 & $\begin{array}{l}\text { F: CCAATCTCGCACTTCTACTCCTCATG } \\
\text { R: ATCGACAGGGTCCTACCTCAATGG }\end{array}$ & $102 \mathrm{bp}$ \\
\hline EGR_07244 & $\begin{array}{l}\text { F:TCCTCCTCGTGGTCATCAGCTATTC } \\
\text { R: CCACAACCGCCGTAGATGAACTC }\end{array}$ & $148 \mathrm{bp}$ \\
\hline EGR_07944 & $\begin{array}{l}\text { F: AAGTGCCTTCATCGCCTCCATTG } \\
\text { R: CCGCCTCTGATTCTGCTTTCTCTG }\end{array}$ & $96 \mathrm{bp}$ \\
\hline EGR_09490 & $\begin{array}{l}\text { F: CCAGTCATAGCGGGCATCAGTTG } \\
\text { R: GTCATCGTCTCCGTGGCATTCC }\end{array}$ & $113 \mathrm{bp}$ \\
\hline EmuJ_001137000 & $\begin{array}{l}\text { F: CTCCTCATGCTGCTCGGTGTTG } \\
\text { R: ATCGACAGGGTCCTACCTCAATGG }\end{array}$ & $85 \mathrm{bp}$ \\
\hline EmuJ_000534800 & $\begin{array}{l}\text { F: TTTCCTCCTTGTGGTCATCAGCTATTC } \\
\text { R: CACAACCGCCGTAGATGAACTCC }\end{array}$ & $149 \mathrm{bp}$ \\
\hline EmuJ_000302900 & $\begin{array}{l}\text { F:TACAATGGTCACGGCGGCAATG } \\
\text { R:TCTGGCGTTCTGGCACAAATCG }\end{array}$ & $99 \mathrm{bp}$ \\
\hline EmuJ_000929500 & $\begin{array}{l}\text { F: GCACATCTTCAGTGGCAGGTATCC } \\
\text { R:TGGCGGTTGGCATTTGGACTAC }\end{array}$ & $87 \mathrm{bp}$ \\
\hline Eif3 & $\begin{array}{l}\text { F: GTTACATCCCTCCGACCTTG } \\
\text { R: AAGCAGCCTCCTCTTGAGTG }\end{array}$ & $243 \mathrm{bp}$ \\
\hline
\end{tabular}

Note: $F$, forward primer; $R$, reverse primer

ratio of absorbance at $260 \mathrm{~nm}$ and $280 \mathrm{~nm}(\mathrm{~A} 260 / 280)$ is ranged from 1.9 to 2.0 .

Total RNA was reverse transcribed into cDNA using Reverse Transcriptase Kit (Takara, Dalian, China) following the instructions of manufacturer. The cDNAs were stored at $-80^{\circ} \mathrm{C}$ until used.

\section{Quantitative PCR (qPCR)}

Gene-specific primers were designed with Primer 3 software and listed in Table 4, eif 3 was used as the internal reference [35].The qPCR reactions were carried out in a CFX96 Real-Time PCR Detection System (BioRad, USA). Experiments were performed with QuantiNova SYBR Green PCR Kit (QIAGEN). Each individual sample was run in triplicate. The qPCR cycling reactions were initially denatured at $95{ }^{\circ} \mathrm{C}$ for $2 \mathrm{~min}$ followed by 39 cycles of $95{ }^{\circ} \mathrm{C}$ for $5 \mathrm{~s}, 60{ }^{\circ} \mathrm{C}$ for $30 \mathrm{~s}$. The melting curve analysis of $5 \mathrm{~s}$ per step from 65 to $95{ }^{\circ} \mathrm{C}$ after amplification was conducted to assess primer specificity. The mRNA expression level of the target cytokines relative to the reference gene were analyzed using the $2^{-\Delta \Delta C t}$ method [36].

\section{Statistical analysis}

Data are presented as means or median. All data are presented as the means \pm standard deviation (SD) of three individual experiments unless otherwise stated. Group comparisons were assessed by Two-tailed Student's t test, Mann-Whitney U test and one-way analysis of variance (ANOVA) for statistically significant differences using GraphPad Prism software (Version 8.01). Chi square test followed by Fisher's Exact Test was used to compare the sample rate (or constituent ratio) between the two groups. $P$ values of $<0.05$ was considered significant in statistical analysis. (* $P$ value $\leq 0.05$; ** $P$ value $\leq 0.001 ; *$ *** $P$ value $\leq 0.0001)$.

\section{Supplementary Information}

The online version contains supplementary material available at https://doi. org/10.1186/s12864-021-08219-4.

Additional file 1: Table S1. Physiological andbiological characteristics of each of E.granulosus S.S. and E. multilocularis KDPIs.

Additional file 2: Table S2. The cellular localization of the E. multilocularis and E. granulosus s.S. KDPIs.

Additional file 3: Figure S1. Structure and amino acidcomposition of a Kunitz-domain peptide.

Additional file 4: Figure S2. Three dimensional structures of multidomain Kunitz protease inhibitors in E.granulosus s.s. and E. multilocularis using SWISS-MODEL.

Additional file 5: Figure S3. Species-specific sequence alignment of $E$. granulosus S.S. and E.multilocularis KDPIs genes.

Additional file 6: Figure S4. Phylogram constructed using the maximum likelihood method to compare EGR_03480 of E. granulosus s.S. with the KDPIs from bovine, humans and other species.

Additional file 7: Figure S5. Phylogenetic analysisof E. granulosus s.S. , E. multilocularis and Fasciola hepatica Kunitz-type inhibitors.

Additional file 8: Figure S6. Sequence alignment of E. granulosus S.S. KU1 8 and Corresponding homologous E. multilocularis KDPIs genes.

Additional file 9: Table S3. The assembly accession numbers of the E.multilocularis, E. granulosus S.S., Fasciolahepatica and Schistosomamansoni $\mathrm{KDPIS}$

Acknowledgements

The authors thank Chuanchuan Wu for his help in producing the figures.

\section{Authors' contributions}

$\mathrm{HZ}$ and MXT participated in biological and physiological characteristic and phylogenetic analysis. MXT, WJQ and JW participated in KDPI sequence confirmation and $3 \mathrm{D}$ analysis. HJZ, GG and LZ participated in InterProScan and Motif scan, expression and GO analysis. JL and WBZ planned the experiments and wrote the article. SLR and DPM were involved in the discussion the article. All authors read and approved the final manuscript.

Funding

This study was funded by the National Natural Science Foundation of China (grant numbers 81830066 and U1803282).

Availability of data and materials

All data generated or analyzed during this study are included in this published article and the additional data file.

\section{Declarations}

Ethics approval and consent to participate not applicable.

Consent for publication

Not applicable. 


\section{Competing interests}

The authors declare no competing interests.

\section{Author details}

${ }^{1}$ Basic Medicine College, Xinjiang Medical University, 830011 Urumqi, Xinjiang, China. ${ }^{2}$ State Key Laboratory of Pathogenesis, Prevention and Treatment of High Incidence Diseases in Central Asia, WHO-Collaborating Centre for Prevention and Care Management of Echinococcosis, Xinjiang Medical University, The First Affiliated Hospital of Xinjiang Medical University, 830054 Urumqi, Xinjiang, China. ${ }^{3}$ Shanghai-Ministry of Science and Technology Key Laboratory of Health and Disease Genomics, Chinese National Human Genome Center at Shanghai, Shanghai, China. ${ }^{4}$ Molecular Parasitology Laboratory, Infectious Diseases Program, QIMR Berghofer Medical Research Institute, Brisbane, Queensland, Australia.

Received: 11 March 2021 Accepted: 11 November 2021 Published online: 18 December 2021

\section{References}

1. Wen H, Vuitton L, Tuxun T, Li J, Vuitton DA, Zhang W, McManus DP. Echinococcosis: Advances in the 21st Century. Clin Microbiol Rev. 2019:32(2):e00075-18.

2. Larrieu E, Gavidia CM, Lightowlers MW. Control of cystic echinococcosis: Background and prospects. Zoonoses Public Health. 2019; 66:889-99.

3. Craig PS, Hegglin D, Lightowlers MW, Torgerson PR, Wang Q. Echinococcosis: Control and Prevention. Adv Parasitol. 2017; 96:55-158.

4. Wa ZC, Du T, Hu HT, Lu MD. Microwave ablation combining surgery for the treatment of multiorgan cystic echinococcosis: A case report. Parasitol Int. 2020; 74:101921.

5. Lopez-Otin C, Bond JS. Proteases: multifunctional enzymes in life and disease. J Biol Chem. 2008; 283:30433-7.

6. Ranasinghe S, McManus DP. Structure and function of invertebrate Kunitz serine protease inhibitors. Dev Comp Immunol. 2013; 39:219-27.

7. Ascenzi P, Bocedi A, Bolognesi M, Spallarossa A, Coletta M, De Cristofaro R, Menegatti E. The bovine basic pancreatic trypsin inhibitor (Kunitz inhibitor): a milestone protein. Curr Protein Pept Sci. 2003; 4:231-51.

8. Rawlings ND, Tolle DP, Barrett AJ. Evolutionary families of peptidase inhibitors. Biochem J. 2004; 378:705-16.

9. Mishra M. Evolutionary Aspects of the Structural Convergence and Functional Diversification of Kunitz-Domain Inhibitors. J Mol Evol. 2020; 88:537-48.

10. Durani V, Magliery TJ. Protein engineering and stabilization from sequence statistics: variation and covariation analysis. Methods Enzymol. 2013: 523:237-56

11. Rustgi S, Boex-Fontvieille E, Reinbothe C, von Wettstein D, Reinbothe S. The complex world of plant protease inhibitors: Insights into a Kunitztype cysteine protease inhibitor of Arabidopsis thaliana. Commun Integr Biol. 2018; $11: \mathrm{e} 1368599$

12. Ranasinghe SL, Fischer K, Gobert GN, McManus DP. Functional expression of a novel Kunitz type protease inhibitor from the human blood fluke Schistosoma mansoni. Parasit Vectors. 2015; 8:408.

13. Ranasinghe SL, Duke M, Harvie M, McManus DP. Kunitz-type protease inhibitor as a vaccine candidate against schistosomiasis mansoni. Int Infect Dis. 2018; 66:26-32.

14. Ranasinghe SL, Fischer K, Zhang W, Gobert GN, McManus DP. Cloning and Characterization of Two Potent Kunitz Type Protease Inhibitors from Echinococcus granulosus. PLoS Negl Trop Dis. 2015; 9:e0004268.

15. Gonzalez S, Flo M, Margenat M, Duran R, Gonzalez-Sapienza G, Grana M, et al. A family of diverse Kunitz inhibitors from Echinococcus granulosus potentially involved in host-parasite cross-talk. PLoS One. 2009; 4:e7009.

16. Krowarsch D, Dadlez M, Buczek O, Krokoszynska I, Smalas AO, Otlewski $J$. Interscaffolding additivity: binding of $\mathrm{P} 1$ variants of bovine pancreatic trypsin inhibitor to four serine proteases. J Mol Biol. 1999; 289:175-86.

17. Grzesiak A, Helland R, Smalas AO, Krowarsch D, Dadlez M, Otlewski J. Substitutions at the $\mathrm{P}(1)$ position in BPTI strongly affect the association energy with serine proteinases. J Mol Biol. 2000; 301:205-17.

18. Zheng H, Zhang W, Zhang L, Zhang Z, Li J, Lu G, et al. The genome of the hydatid tapeworm Echinococcus granulosus. Nat Genet. 2013; 45:1168-75.
19. de Magalhaes MTQ, Mambelli FS, Santos BPO, Morais SB, Oliveira SC. Serine protease inhibitors containing a Kunitz domain: their role in modulation of host inflammatory responses and parasite survival. Microbes Infect. 2018; 20:606-9.

20. Ranasinghe SL, Fischer K, Gobert GN, McManus DP. A novel coagulation inhibitor from Schistosoma japonicum. Parasitology. 2015; 142:1663-72.

21. Falcon CR, Masih D, Gatti G, Sanchez MC, Motran CC, Cervi L. Fasciola hepatica Kunitz type molecule decreases dendritic cell activation and their ability to induce inflammatory responses. PLoS One. 2014; 9:e114505.

22. Cwiklinski K, de la Torre-Escudero E, Trelis M, Bernal D, Dufresne PJ, Brennan GP, et al. The Extracellular Vesicles of the Helminth Pathogen, Fasciola hepatica: Biogenesis Pathways and Cargo Molecules Involved in Parasite Pathogenesis. Mol Cell Proteomics. 2015; 14:3258-73.

23. Kooyman FN, van Balkom BW, de Vries E, van Putten JP. Identification of a thrombospondin-like immunodominant and phosphorylcholinecontaining glycoprotein (GP300) in Dictyocaulus viviparus and related nematodes. Mol Biochem Parasitol. 2009; 163:85-94.

24. Milstone AM, Harrison LM, Bungiro RD, Kuzmic P, Cappello M. A broad spectrum Kunitz type serine protease inhibitor secreted by the hookworm Ancylostoma ceylanicum. J Biol Chem. 2000; 275:29391-9.

25. Toubarro D, Avila MM, Montiel R, Simoes N. A pathogenic nematode targets recognition proteins to avoid insect defenses. PLoS One. 2013; 8:e75691.

26. Khrustalev W. Random Coils of Proteins Situated Between a Beta Strand and an Alpha Helix Demonstrate Decreased Solvent Accessibility. Protein J. 2020: 39:308-17.

27. Creighton TE. The two-disulphide intermediates and the folding pathway of reduced pancreatic trypsin inhibitor. J Mol Biol. 1975; 95:167-99.

28. Laskowski M, Jr., Kato I. Protein inhibitors of proteinases. Annu Rev Biochem. 1980; 49:593-626.

29. Flo M, Margenat M, Pellizza L, Grana M, Duran R, Baez A, et al. Functional diversity of secreted cestode Kunitz proteins: Inhibition of serine peptidases and blockade of cation channels. PLoS Pathog. 2017; 13:e1006169.

30. Jedlickova L, Dvorak J, Hrachovinova I, Ulrychova L, Kasny M, Mikes L. A novel Kunitz protein with proposed dual function from Eudiplozoon nipponicum (Monogenea) impairs haemostasis and action of complement in vitro. Int J Parasitol. 2019; 49:337-46.

31. Silvane L, Celias DP, Romagnoli PA, Maletto BA, Sanchez Vallecillo MF, Chiapello LS, et al. A Vaccine Based on Kunitz-Type Molecule Confers Protection Against Fasciola hepatica Challenge by Inducing IFN-gamma and Antibody Immune Responses Through IL-17A Production. Front Immunol. 2020; 11:2087.

32. Hernandez-Goenaga J, Lopez-Aban J, Protasio AV, Vicente Santiago B, Del Olmo E, Vanegas M, Fernandez-Soto P, Patarroyo MA, Muro A. Peptides Derived of Kunitz-Type Serine Protease Inhibitor as Potential Vaccine Against Experimental Schistosomiasis. Frontiers in immunology. 2019; 10:2498.

33. Smith D, Cwiklinski K, Jewhurst H, Tikhonova IG, Dalton JP. An atypical and functionally diverse family of Kunitz-type cysteine/serine proteinase inhibitors secreted by the helminth parasite Fasciola hepatica. Sci Rep. 2020; 10:20657.

34. Tsai IJ, Zarowiecki M, Holroyd N, Garciarrubio A, Sanchez-Flores A, Brooks $\mathrm{KL}$, et al. The genomes of four tapeworm species reveal adaptations to parasitism. Nature. 2013; 496:57-63.

35. Zhang W, Li J, Jones MK, Zhang Z, Zhao L, Blair D, McManus DP. The Echinococcus granulosus antigen $B$ gene family comprises at least 10 unique genes in five subclasses which are differentially expressed. PLoS Negl Trop Dis. 2010; 4:e784.

36. Livak KJ, Schmittgen TD. Analysis of relative gene expression data using real-time quantitative PCR and the 2(-Delta Delta C(T)) Method. Methods. $2001 ; 25: 402-8$.

\section{Publisher's Note}

Springer Nature remains neutral with regard to jurisdictional claims in published maps and institutional affiliations. 